\title{
Fractionated radiosurgery for painful spinal metastases: DOSIS - a phase II trial
}

Matthias Guckenberger ${ }^{1 *}$, Maria Hawkins ${ }^{2}$, Michael Flentje ${ }^{1}$ and Reinhart A Sweeney ${ }^{1}$

\begin{abstract}
Background: One third of all cancer patients will develop bone metastases and the vertebral column is involved in approximately $70 \%$ of these patients. Conventional radiotherapy with of 1-10 fractions and total doses of 8-30 Gy is the current standard for painful vertebral metastases; however, the median pain response is short with 3-6 months and local tumor control is limited with these rather low irradiation doses. Recent advances in radiotherapy technology - intensity modulated radiotherapy for generation of highly conformal dose distributions and image-guidance for precise treatment delivery - have made dose-escalated radiosurgery of spinal metastases possible and early results of pain and local tumor control are promising. The current study will investigate efficacy and safety of radiosurgery for painful vertebral metastases and three characteristics will distinguish this study. 1) A prognostic score for overall survival will be used for selection of patients with longer life expectancy to allow for analysis of long-term efficacy and safety. 2) Fractionated radiosurgery will be performed with the number of treatment fractions adjusted to either good (10 fractions) or intermediate (5 fractions) life expectancy. Fractionation will allow inclusion of tumors immediately abutting the spinal cord due to higher biological effective doses at the tumor - spinal cord interface compared to single fraction treatment. 3) Dose intensification will be performed in the involved parts of the vertebrae only, while uninvolved parts are treated with conventional doses using the simultaneous integrated boost concept.
\end{abstract}

Methods / Design: It is the study hypothesis that hypo-fractionated image-guided radiosurgery significantly improves pain relief compared to historic data of conventionally fractionated radiotherapy. Primary endpoint is pain response 3 months after radiosurgery, which is defined as pain reduction of $\geq 2$ points at the treated vertebral site on the 0 to 10 Visual Analogue Scale. 60 patients will be included into this two-centre phase II trial.

Conclusions: Results of this study will refine the methods of patient selection, target volume definition, treatment planning and delivery as well as quality assurance for radiosurgery. It is the intention of this study to form the basis for a future randomized controlled trial comparing conventional radiotherapy with fractionated radiosurgery for palliation of painful vertebral metastases.

Trial registration: ClinicalTrials.gov Identifier: NCT01594892

Keywords: Phase II trial, Spinal metastasis, Pain, Radiosurgery, Stereotactic body radiotherapy

\footnotetext{
* Correspondence: Guckenberger_M@klinik.uni-wuerzburg.de

1 Department of Radiation Oncology, University of Würzburg,

Josef-Schneider-Str. 11, Würzburg 97080, Germany

Full list of author information is available at the end of the article
} 


\section{Background}

\section{Conventional palliative radiotherapy for painful vertebral} metastases

Approximately one third of all patients with cancer will develop bone metastases [1] and of these patients, approximately $70 \%$ will have metastases involving the vertebral column, most commonly the thoracic and lumbar spine. Radiation therapy plays an important role in the multidisciplinary treatment of symptomatic vertebral metastases, including palliation of pain, control or prevention of neurological symptoms, and prevention of pathologic fractures.

During the past three decades, the gold standard of radiotherapy for painful bony metastases has been based on several randomized trials comparing various radiotherapy fractionation schemas: the majority of the studies compared single fraction radiotherapy of 8 Gy with fractionated protocols of 5-10 fractions and total doses of 20-30 Gy (meta-analyses in [2,3]). Radiotherapy was effective with overall pain relief in $70 \%$ of the patients on average. No dose-response relationship has been demonstrated in the meta-analyses meaning that increased irradiation doses delivered in multiple fractions did not result in higher pain response rates than a single fraction of $8 \mathrm{~Gy}$ and this palliative regime is consequently the evidence-based standard of care [4].

Nevertheless, there are clinical data suggesting that patients with painful vertebral metastases might benefit from higher irradiation doses than used in the prospective trials cited above. Despite overall pain response being high at $70 \%$, the median duration of pain response is only $3-6$ months. Consequently, only approximately $1 / 3$ of the patients are effectively palliated for a duration of a few months with these conventional radiotherapy schemas. Additionally, partial pain response should not be the primary goal of treatment but rather complete pain response, which is achieved in only one quarter of the patients.

Studies on metastatic spinal cord compression (MSCC) confirm that conventional irradiation doses might not be sufficient for intermediate or even long term local disease control. Patchell et al. reported a randomized trial comparing radiotherapy alone with decompressive surgery followed by radiotherapy [5]; radiotherapy consisted of ten fractions of 3 Gy. Maintenance of walking ability was achieved for a median duration of 13 days and 122 days in the radiotherapy alone arm compared to combined treatment indicating that intensification of local treatment improved the outcome. Another randomized study compared lower and higher dose radiotherapy for MSCC [6]. Whereas no difference in response rates was observed for the total patient population, significantly improved response after high dose compared to low dose irradiation was observed in the subgroup of patients with unfavourable histologies (lung, kidney, gastrointestinal, head and neck, melanoma and sarcoma); a dose response relationship was not observed in patients with favourable histologies (lymphoma, seminoma, breast, prostate, myeloma). This again indicates that subgroups of patients might benefit from intensified local radiotherapy in this palliative setting.

Diagnostic possibilities and systemic treatments have significantly evolved in the recent years, with many patients surviving a metastatic state for years, which increases the need for more effective treatment of spinal metastases. Furthermore, prognostic scoring systems are available, which allow selection of patients with favourable traits [5-7]. These patients with life expectancy longer than $3-6$ months might benefit from or even require a radiotherapy treatment offering more durable pain control, maintenance of quality-of-life and of neurological function.

\section{Radiosurgery for painful vertebral metastases}

Stereotactic radiosurgery for intracranial metastases delivered either with or without whole brain radiation therapy, has been associated with very high rates of local tumour control, in the range of $85-95 \%$ across studies [7-12]. This stereotactic radiosurgery not only improved local tumor control but also overall survival when practiced in patients with a solitary brain metastasis. These results, in conjunction with very low complication rates related to the highly conformal nature of the treatment, have made intracranial radiosurgery an increasingly popular and available treatment modality.

Irradiation with such escalated "radiosurgical" doses has not been possible for spinal metastases because of the anatomical situation, where the tumor is very close to the spinal cord and frequently even wrapped around this critical organ-at-risk. Traditional technologies made it impossible to achieve a sufficiently high irradiation dose in this complex shaped target volume while simultaneously keeping the dose to the spinal cord within accepted tolerances [13]. Two recent advances in radiotherapy technologies have made it possible to transfer the radiosurgical concept from the brain to the vertebral region. Intensity-modulated radiotherapy (IMRT) allows the generation of convexly shaped dose distributions sparing the spinal cord from high irradiation doses. Image-guided (IGRT) verification of patient set-up enables accurate delivery of the planned dose distributions, which is especially important from a safety perspective because of the steep dose gradients between the target and the spinal cord.

In a recent survey, more than $40 \%$ of all radiation oncologists in the US stated that they practice spine radiosurgery and most institutions favour single-fraction radiosurgical techniques due to best patient comfort 
with a "one-shot" outpatient treatment in the context of limited life expectancy [14]. Rapid adoption of spinal radiosurgery is observed despite the paucity of prospective trials. Only one prospective study has been published so far [15] and the randomized RTOG 0631 is still accruing patients. Nevertheless, results of these radiosurgical studies are promising: local tumor control / pain control has been reported in $80-90 \%$ of the patients and this was achieved with low rates of severe toxicity [15-20]. The most serious toxicity - radiation induced myelopathy - has been reported in less than $1 \%$ of the patients in a large analysis of $>1000$ treatments [21].

The concept of radiosurgery practiced as single fraction, however, has limitations. Due to the immediate proximity of vertebral metastases to the spinal cord, the treatment dose deliverable is limited by the tolerance of the spinal cord [21-24]. In cases of radiosurgical treatment of these spinal cord abutting tumors, the epidural tumor component has been identified as the most frequent site of treatment failure: both the spinal cord and adjacent epidural tumour involvement were spared from high biological doses of radiosurgery [16-18,25,26]. Consequently, many study protocols exclude tumors within a distance of $<3 \mathrm{~mm}$ to the spinal cord, as does the RTOG 0631 trial. This however precludes exactly those patients which are at the highest risk of cord compression.

\section{Rationale for this trial}

The rationale for this trial is based on the same hypothesis as the single-fraction radiosurgical studies: to improve pain control and local tumor control via dose intensified radiotherapy for vertebral metastases. This trial will differ in three important aspects from the currently available literature and trials:

1) A prognostic score for overall survival (modified Mizumoto Score) will be used for selection of patients with favourable life expectancy [27], who are expected to benefit most from the intensified radiotherapy. The number for treatment fractions will be adjusted based on the modified Mizumoto Score to balance intensity and length of treatment with life expectancy. Selection of patients with long life expectancy will also allow for evaluation of late toxicity and long-term efficacy.

2) Hypo-fractionated radiosurgery with 5 and 10 treatment fractions will be practiced in this study. Based on radiobiological modelling, hypofractionated radiosurgery allows higher biologically effective doses to the tumor directly adjacent to the spinal cord than single-fraction protocols.

Recurrences at the interface between the spinal cord and the epidural tumor are expected to be reduced by fractionation. This will expand the indications for dose intensified radiotherapy to tumors immediately abutting the spinal cord.

3) Failures in untreated parts of the vertebrae shall be avoided by the use of a simultaneous integrated boost concept. Similar to the single-fraction radiosurgical studies, dose intensification will be performed in the macroscopic tumour using dedicated CT and MR imaging. However, the noninvolved parts of the vertebra will be treated with a second dose level (conventional dose) to avoid recurrences in the untreated parts of the vertebrae.

Preliminary results from the University Hospital Wuerzburg with dose intensified irradiation using 20 fractions of 3 Gy [28] are promising and support the hypothesis of this trial; Patients with long life expectancy were selected for fractionated radiosurgery resulting in an actuarial local control rate and overall survival of $88 \%$ and $63 \%$ after 2 years [13]. No acute or chronic toxicity $>$ Grade 2 was seen.

\section{Methods/design of the trial Study design}

The study is designed as a prospective phase II trial. The outline of the study protocol is shown in Figure 1.

\section{Study hypothesis}

Hypo-fractionated image guided radiosurgery significantly improves pain relief compared to historic data of conventionally fractionated radiotherapy.

\section{Primary endpoint}

The primary objective is to determine whether a sustained pain relief can be achieved with dose intensified hypo-fractionated image-guided radiosurgery in patients with vertebral metastasis and intermediate and long life expectancy based on the modified Mizumoto Score. The Mizumoto score is slightly modified to improve overall survival in the group with intermediate life expectancy. Pain response 3 months after radiosurgery will be evaluated as primary endpoint and pain reduction of $\geq 2$ points at the treated vertebral site on the 0 to 10 Visual Analogue Scale without analgesic increase will be defined as pain response [29].

\section{Secondary endpoints}

1. Local tumor control at the treated vertebral levels and regional tumor control at the neighbouring vertebrae

2. Overall survival and cancer specific mortality

3. Quality of life using the EQ-5D and EORTC QLQ-BM22

4. Acute and late toxicity according to NCI CTCAE v 4.0 


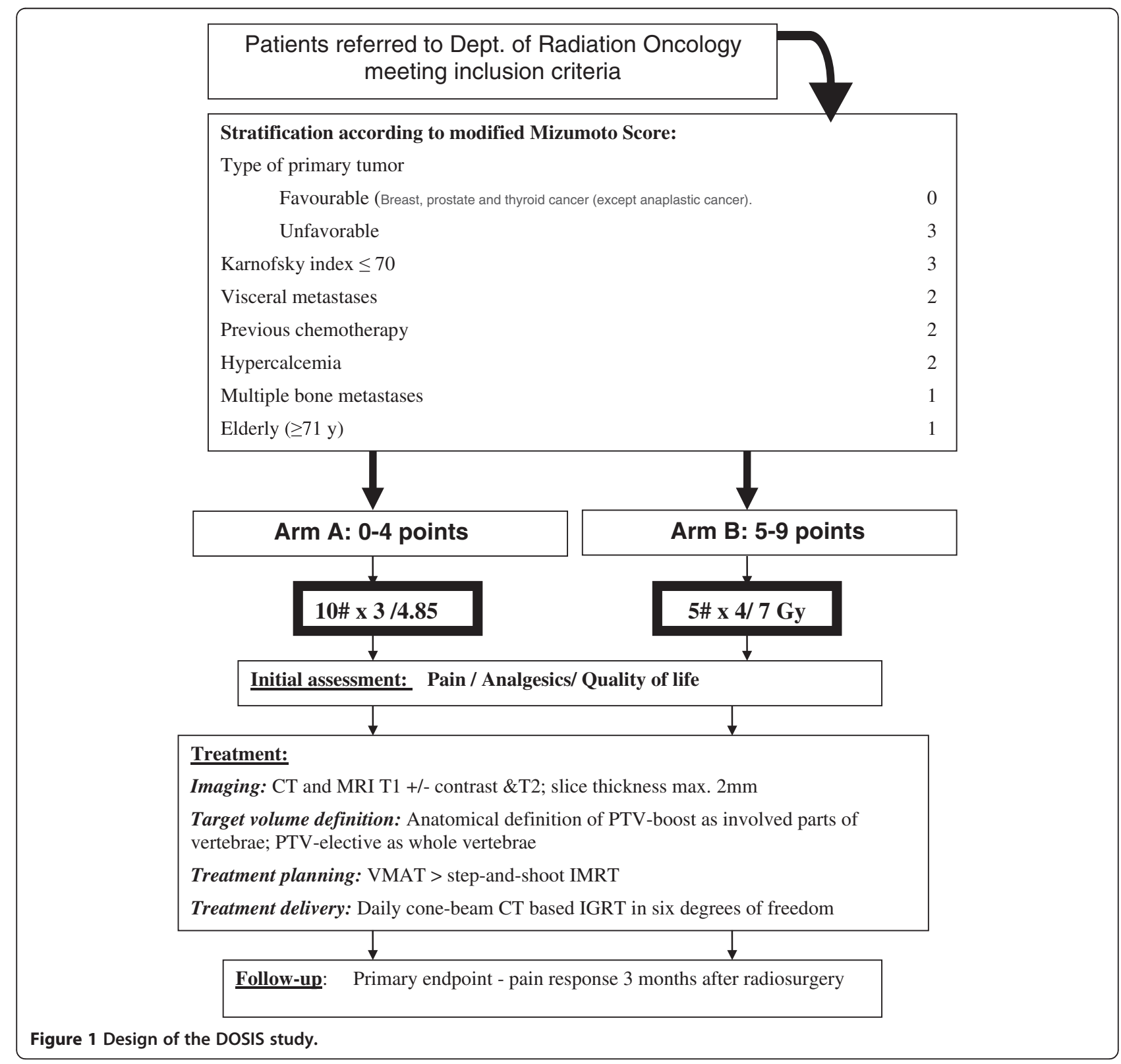

\section{Exploratory endpoints}

1. Time between initial consult and first treatment (the goal is to reduce this period to within 48 hours by maximizing use of standardized procedures of diagnostics, fixation and planning)

2. Repositioning and image-guidance accuracy within the fixation system in six degrees of freedom

3. Intra-fraction motion as analysed by comparison of cone-beam CT imaging immediately preceding treatment

4. Morphological pattern of CT and MRI radiological response of vertebral metastases after doseintensified, hypo-fractionated radiosurgery

\section{Inclusion criteria}

Patients with the following characteristics will be eligible for this study

1. Established histological diagnosis of a malignant tumour (primary or metastatic)

2. Vertebral metastasis confirmed via biopsy or radiology

3. Pain in the involved spinal region or free of pain under pain medication

4. Fully consenting patients, $>18$ years old

5. Karnofsky Performance Index $\geq 60 \%$

6. Good or intermediate life expectancy according to the modified prognostic Mizumoto Score $($ score $\leq 9)$ 
7. Patient must be able to tolerate fixation systems and 30 minutes treatment time

8. Discussed in interdisciplinary tumour board

9. The following types of spinal tumours are eligible:

- Recurrent / residual tumours after surgery

- Tumours in medically inoperable patients or patients deemed inoperable due to limited life expectancy / tumour load

- Lesions associated with significant surgical risk

\section{Exclusion criteria}

Patients with the following characteristics will be ineligible for this study

1. Short life expectancy according to the modified Mizumoto Sore

2. "Radiosensitive" histologies (i.e. lymphoma, SCLC, multiple myeloma)

3. Non-ambulatory status

4. Progressive neurological symptoms/deficit

5. $>3$ involved vertebral levels

6. $>2$ treatment sites

7. Spine instability

8. Previous radiotherapy at the involved levels

\section{Treatment planning}

\section{Patient positioning and imaging}

No specific pre-medication will be applied. The patient will be placed in a stable position in the immobilization device (such as Body Fix). For cervical spine lesions, a combination of BodyFix and thermoplastic mask will be used. All patients will undergo a treatment planning CT scan immobilized in the treatment position with reconstruction of $2 \mathrm{~mm}$ axial slices. 3D volume imaging will be used for MRI imaging with $0.8-2 \mathrm{~mm}$ axial slices. T1 pre- and post-contrast as well as T2 sequences are required. The respective MRI scans will be registered with the treatment planning $\mathrm{CT}$ scan using rigid image registration.

\section{Target and organs-at-risk delineation}

The target volume concept is illustrated in Figure 2.

Gross tumour volume (GTV): The target lesion will be outlined on the planning MRI (GTV-MRI) and in cases without MRI imaging on the treatment planning $\mathrm{CT}$ scan (GTV-CT).

Planning target volume boost (PTV-boost): The PTVboost will be based on an anatomical target volume concept, where all macroscopically involved elements of the involved vertebra (body, pedicles, transverse process, spinous process) are defined as PTV-boost.

PTV-elective: Defined as the entire vertebrae of the involved levels.
Organs at risk: The spinal cord will be contoured according to the MRI. A $1 \mathrm{~mm}$ expansion of the spinal cord will be performed to account for set-up errors, this being the spinal cord OAR. Additional organs-at-risk to be delineated are pharynx, oesophagus, lungs, kidneys and bowel.

\section{Treatment planning}

Patients will be treated with a linear accelerator with beam energies varying form 6-18MV. Inversely optimized intensity-modulated radiotherapy will be mandatory. Volumetric modulated arc therapy (VMAT) will be preferably utilized compared to step and shoot IMRT to minimize treatment times.

Two different fractionations will be used depending on estimated life expectancy: patients with long life expectancy (Arm A: Mizumoto score 0-4) will be treated with 10 fractions whereas patients with intermediate life expectancy (Arm B: Mizumoto score 5-9) will be treated with only 5 fractions. Total treatment doses will be 30 Gy and 48.5 Gy at the "PTV-elective" and at the "PTV-boost" for Arm A, respectively. Total treatment doses will be 20 Gy and 35 Gy at the "PTV-elective" and at the "PTV-boost" for Arm B, respectively. Two-Gray equivalent doses $(\alpha / \beta=10$ Gy) at the PTV-boost are 50 Gy and 60 Gy for the 5-fractions and 10-fractions regimen, respectively. The patients will be treated on consecutive workdays, with one fraction per day.

Normal tissue dose constraints are summarized in Table 1.

\section{Treatment delivery}

Volumetric cone-beam CT (CBCT) imaging will be obtained at each treatment after patient positioning to verify patient set-up. Another verification scan will be acquired following on-line correction to ensure a residual error of $\leq 1 \mathrm{~mm}$ and $\leq 1^{\circ}$, and one following beam delivery to assess for intra-fraction drift.

\section{Trial duration and follow-up assessments}

The primary endpoint will be pain control assessed 3 months after radiotherapy. Detailed follow-up is outlined in Table 2. Patient follow-up will continue for five years to evaluate the secondary end points of the trial. Patient accrual is estimated to be finished within 2 years.

For evaluation of pain a 10 point visual analogue scale will be used [15] and analgesic use will be recorded. Pain response is defined as a reduction of $\geq 2$ points at the treated site without analgesic increase. Complete pain response is defined as a pain score of 0 at the treated site with no concomitant increase in analgesic intake. Stable pain is defined as unchanged score or within 2 points of the baseline with no increase in analgesia. 


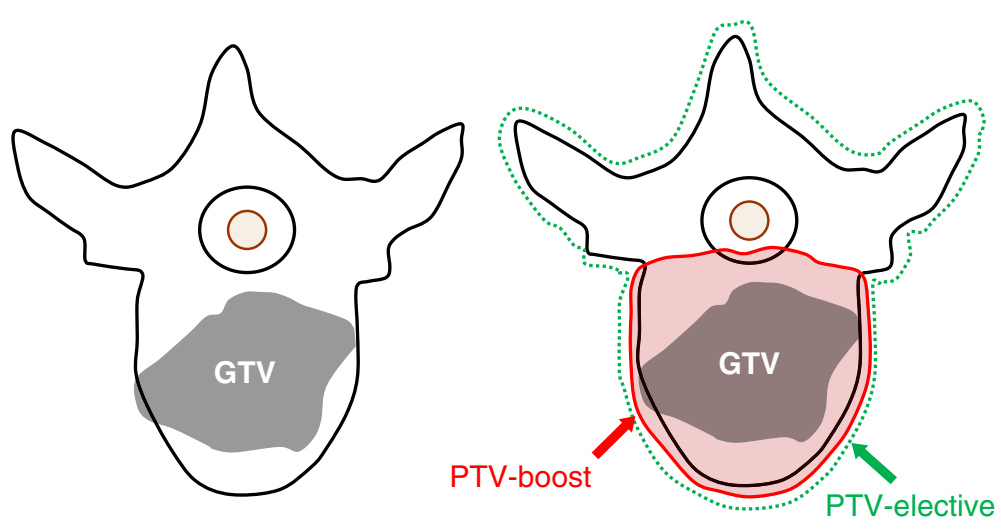

Figure 2 Concept for definition of the PTV-boost and PTV-elective and illustration based on three cases with different GTV locations and macroscopic tumor extensions.

Quality of life will be analysed using the EQ-5D and EORTC QLQ-BM22 questionnaires.

Local control will be defined by repeat imaging (preferably MRT or CT). Progression events are defined as radiological documented disease progression using RECISTCriteria (Revised Guidelines, Version 1.1, 2009) [30].

Kaplan Meier curves for local tumor control, cancer specific survival and overall survival will be calculated starting from the first day of treatment.

This study will use the International Common Terminology Criteria for Adverse Events (CTCAE) version 4.0 for toxicity and adverse event reporting.

\section{Criteria for consideration of study termination}

The trial may be stopped prior to meeting the accrual goal if the toxicity of hypo-fractionated spinal radiosurgery is determined to be unacceptable. Acute or late toxicity will be considered unacceptable if the rate of CTCAE v4.0 grade 3 toxicity is greater than $30 \%$, grade 4 toxicity greater than $20 \%$, or any grade 5 toxicity attributable to therapy occurs. Interim analyses of toxicity will be planned after the first 10 patients have been accrued, after an additional 20 patients, and after the total number of evaluable patients have been accrued to the study with adequate follow-up (generally $>90$ days after completion of treatment) for assessment of toxicity.

\section{Sample size calculation}

This study aims to demonstrate an improvement in pain control at 3 months from approximately $40-50 \%$ with conventionally fractionated radiotherapy to $70-80 \%$ with image-guided, hypo-fractionated radiotherapy. Assuming an improvement in pain control from $45 \%$ to $75 \%$ sample size calculations at a $5 \%$ statistical significant level show that 27 patients would be required to achieve power of the study of $90 \%$. This is the total number of patients eligible for statistical analysis per study arm, i.e. 54 in total. Considering a $10 \%$ drop-out rate, the total number of patients is 60 . No comparison will be made between the two study arms.

\section{Ethical and legal considerations}

The DOSIS study is conducted in line with either the Declaration of Helsinki (Tokyo, Venice, Hong Kong, Somerset West and Edinburgh amendments) or the laws and regulations of the country, whichever provides the greatest protection of the patient. The protocol has been written, and the study will be conducted according to

Table 1 Organ-at-risk dose constraints

\begin{tabular}{|c|c|c|c|c|c|}
\hline & \multicolumn{2}{|c|}{ Maximum dose } & \multirow[b]{2}{*}{ Volume of max dose } & \multicolumn{2}{|c|}{ Mean Dose } \\
\hline & Arm B & Arm A & & Arm B & Arm A \\
\hline Fractionation Scheme & $5 \times 4 / 7$ & $10 \times 3 / 4.85$ & & $5 \times 4 / 7$ & $10 \times 3 / 4.85$ \\
\hline Spinal Cord $+1 \mathrm{~mm}$ & 23.75 & 35 & $0.1 \mathrm{~cm}^{3}$ & & \\
\hline Cauda Equina & 25 & 37.5 & $0.1 \mathrm{~cm}^{3}$ & & \\
\hline Kidney & - & - & & 10 & 12 \\
\hline Bowel & 24 & 37 & $1 \mathrm{~cm}^{3}$ & - & - \\
\hline Esophagus & 30 & 40 & $1 \mathrm{~cm}^{3}$ & - & - \\
\hline Liver & & - & & 12.5 & 17.5 \\
\hline
\end{tabular}

Normal tissue constraints depending upon the fractionation schema. 
Table 2 Patient follow-up and assessment scheme

\begin{tabular}{|c|c|c|c|c|c|c|c|c|c|c|c|}
\hline Assessment & Prior RT & $\begin{array}{l}\text { weekly } \\
\text { during } \mathrm{RT}\end{array}$ & Post RT & 1-4 weeks & 6 weeks & 3 mon & 6 mon & 9 mon & 12 mon & 18 mon & 24 mon \\
\hline Physical Examination & $x$ & $x$ & $x$ & & $x$ & $x$ & $x$ & & $x$ & & $x$ \\
\hline Karnofsky performance index & $x$ & $x$ & $x$ & & $x$ & $x$ & $x$ & & $x$ & & $x$ \\
\hline $\begin{array}{l}\text { Documentation of } \\
\text { Pain \& Analgesic Use }\end{array}$ & $x$ & $x$ & $x$ & $\begin{array}{l}\text { Self } \\
\text { assessment }\end{array}$ & $x$ & $x$ & $x$ & Tel. & $x$ & Tel. & $x$ \\
\hline EQ-5D & $x$ & $x$ & $x$ & & $x$ & $x$ & $x$ & & $x$ & & $x$ \\
\hline QLQ-BM22 & $x$ & $x$ & $x$ & & $x$ & $x$ & $x$ & & $x$ & & $x$ \\
\hline $\mathrm{NCI}$ CTCAE v4 toxicity & & $x$ & $x$ & & $x$ & $x$ & $x$ & & $x$ & & $x$ \\
\hline MRI / CT imaging & $x$ & & & & $x$ & $x$ & $x$ & & $x$ & & $X$ \\
\hline
\end{tabular}

Tel: patients will be interrogated by a study nurse via telephone.

Self-assessment: patients will be given letters for self-assessment of pain and analgetic use for the four weeks following radiosurgery.

the ICH Harmonized Tripartite Guideline for Good Clinical Practice (http://www.ifpma.org/pdfifpma/e6.pdf).

The trial protocol, patient information and informed consent sheets have been approved by the independent ethics committee of the University Hospital, University of Wuerzburg and the ethics committees of the Royal Marsden Hospital NHS Foundation Trust.

The study protocol has been reviewed by the "Independent Expert Committee of the DEGRO" and was considered as application of therapeutic radiotherapy within the frame of current health care.

\section{Sponsorship}

For patients treated at the Royal Marsden Hospital, the study sponsor is the Royal Marsden NHS Foundation Trust, Downs Road, Sutton, Surrey, SM2 5PT, United Kingdom. For patients treated at the Department of Radiation Oncology of the University Hospital Wuerzburg, the study sponsor is the University Hospital Wuerzburg, Wuerzburg, Germany.

\section{Discussion}

This study will assess efficacy and safety of dose-intensified radiosurgery for painful vertebral metastases. Data from this study will help to model a potential relationship between irradiation dose and pain palliation. Such a doseresponse relationship has not been demonstrated in randomized trials before: we hypothesize that irradiation doses in all arms of the randomized trials were in the flat region of the sigmoid-shaped dose-response curve and higher doses are required to improve clinical results. The modern technologies of IMRT and IGRT, which are mandatory in this study, will be used to realize planning and delivery of such escalated doses safely. Despite pain control at three months being the primary endpoint, patients will remain in follow-up for five years for analysis of long-term pain control, local tumor control and toxicity.
Follow-up was limited in the majority of the existing studies due to short overall survival of unselected patients. Patients with estimated short life expectancy will be excluded from this study, which will allow evaluation of long-term efficacy and in particular safety of intensified radiosurgery in this palliative setting. More reliable data on long-term safety with a particular focus on radiation induced myelopathy is considered highly important for further development of spine radiosurgery.

Fractionated radiosurgery allows the inclusion of patients, whose tumor actually contacts the spinal cord. Fractionation will enable higher biological doses at the target / organ-at-risk interface. This is expected to reduce the local failures, which were most likely the result of underdosing the volumes in this high-risk region. Patients with the metastases approaching the spinal cord but without neurological deficits are not typical surgical candidates and local radiotherapy with durable tumor control might be especially warranted in this patient collective for prevention of MSCC.

Fractionation might also be beneficial from a safety perspective. Despite inter- and intra-fractional patient motion being minimized by IGRT and patient immobilization devices, residual errors need to be considered and cannot fully be avoided. Fractionation is expected to limit the consequences of such errors unless they are systematic and occur on a daily basis. This will be avoided by strict quality assurance.

In conventional radiotherapy for spinal metastases, the involved vertebral levels as well as the two adjacent vertebrae were defined as target volume. This was practiced because of uncertainties of disease extension as well as low accuracy of treatment delivery. We will limit the target volume to the involved vertebrae based on radiosurgical experiences of low recurrence rates in the adjacent noninvolved vertebrae $[17,25,31]$, but regional control in these vertebrae will be a secondary endpoint of this analysis. In contrast to most radiosurgical studies, we will continue 
treating the whole metastatic vertebrae. This will be achieved using the simultaneous integrated boost approach, where dose intensification will be applied to the involved parts of the vertebrae only and uninvolved parts will be treated with conventional doses. It is intended to perform a detailed analysis of recurrences with dose effect modelling, which will help to refine evidence-based target volumes for future trials.

It is the intention of this study to form the basis for a future phase III trial comparing conventional radiotherapy with radiosurgery for palliation of painful vertebral metastases in a randomized manner. The methods of patient selection, target volume definition, treatment planning and delivery as well as quality assurance will be redefined based on the results of this study.

\section{Competing interests}

The authors declare that they have no competing interests.

\section{Authors' contributions}

MG and RAS designed the study and MH and MF participated in the design of the study. All authors performed critical review of the manuscript and finally approved the manuscript.

\section{Acknowledgements}

This publication was funded by the German Research Foundation (DFG) and the University of Wuerzburg in the funding programme Open Access Publishing.

\section{Author details}

${ }^{1}$ Department of Radiation Oncology, University of Würzburg, Josef-Schneider-Str. 11, Würzburg 97080, Germany. ${ }^{2}$ The Royal Marsden Hospital NHS Foundation Trust, London, United Kingdom.

Received: 8 May 2012 Accepted: 10 November 2012

Published: 19 November 2012

\section{References}

1. Wong DA, Fornasier VL, MacNab I: Spinal metastases: the obvious, the occult, and the impostors. Spine 1990, 15(1):1-4.

2. Chow E, Harris K, Fan G, Tsao M, Sze WM: Palliative radiotherapy trials for bone metastases: a systematic review. J Clin Oncol 2007, 25(11):1423-1436.

3. Wu JS, Wong R, Johnston M, Bezjak A, Whelan T: Meta-analysis of dosefractionation radiotherapy trials for the palliation of painful bone metastases. Int J Radiat Oncol Biol Phys 2003, 55(3):594-605.

4. Lutz S, Berk L, Chang E, Chow E, Hahn C, Hoskin P, Howell D, Konski A, Kachnic L, Lo S, et al: Palliative radiotherapy for bone metastases: an ASTRO evidence-based guideline. Int J Radiat Oncol Biol Phys 2011, 79(4):965-976.

5. Patchell RA, Tibbs PA, Regine WF, Payne R, Saris S, Kryscio RJ, Mohiuddin M, Young B: Direct decompressive surgical resection in the treatment of spinal cord compression caused by metastatic cancer: a randomised trial. Lancet 2005, 366(9486):643-648.

6. Maranzano E, Bellavita R, Rossi R, De Angelis V, Frattegiani A, Bagnoli R, Mignogna M, Beneventi S, Lupattelli M, Ponticelli P, et al: Short-course versus split-course radiotherapy in metastatic spinal cord compression: results of a phase III, randomized, multicenter trial. J Clin Oncol 2005, 23(15):3358-3365.

7. Andrews DW, Scott CB, Sperduto PW, Flanders AE, Gaspar LE, Schell MC, Werner-Wasik M, Demas W, Ryu J, Bahary JP, et al: Whole brain radiation therapy with or without stereotactic radiosurgery boost for patients with one to three brain metastases: phase III results of the RTOG 9508 randomised trial. Lancet 2004, 363(9422):1665.

8. Sneed PK, Suh JH, Goetsch SJ, Sanghavi SN, Chappell R, Buatti JM, Regine WF, Weltman E, King VJ, Breneman JC, et al: A multi-institutional review of radiosurgery alone vs. radiosurgery with whole brain radiotherapy as the initial management of brain metastases. Int J Radiat Oncol Biol Phys 2002, 53(3):519.

9. Rades D, Bohlen G, Pluemer A, Veninga T, Hanssens P, Dunst J, Schild SE: Stereotactic radiosurgery alone versus resection plus whole-brain radiotherapy for 1 or 2 brain metastases in recursive partitioning analysis class 1 and 2 patients. Cancer 2007, 109(12):2515-2521.

10. Kondziolka D, Martin JJ, Flickinger JC, Friedland DM, Brufsky AM, Baar J, Agarwala S, Kirkwood JM, Lunsford LD: Long-term survivors after gamma knife radiosurgery for brain metastases. Cancer 2005, 104(12):2784-2791.

11. Mehta MP, Tsao MN, Whelan TJ, Morris DE, Hayman JA, Flickinger JC, Mills M, Rogers CL, Souhami L: The American Society for Therapeutic Radiology and Oncology (ASTRO) evidence-based review of the role of radiosurgery for brain metastases. Int J Radiat Oncol Biol Phys 2005, 63(1):37-46.

12. Hasegawa T, Kondziolka D, Flickinger JC, Germanwala A, Lunsford LD: Brain metastases treated with radiosurgery alone: an alternative to whole brain radiotherapy? Neurosurgery 2003, 52(6):1318-1326. discussion 1326.

13. Schultheiss TE, Kun LE, Ang KK, Stephens LC: Radiation response of the central nervous system. Int J Radiat Oncol Biol Phys 1995, 31(5):1093-1112.

14. Pan H, Simpson DR, Mell LK, Mundt AJ, Lawson JD: A survey of stereotactic body radiotherapy use in the United States. Cancer 2011, 117(19):4566-4572.

15. Wang XS, Rhines LD, Shiu AS, Yang JN, Selek U, Gning I, Liu P, Allen PK, Azeem SS, Brown PD, et al: Stereotactic body radiation therapy for management of spinal metastases in patients without spinal cord compression: a phase 1-2 trial. Lancet Oncol 2012, 13(4):395-402.

16. Ryu S, Rock J, Rosenblum M, Kim JH: Patterns of failure after single-dose radiosurgery for spinal metastasis. J Neurosurg 2004, 101(Suppl 3):402-405.

17. Gerszten PC, Burton SA, Ozhasoglu C, Welch WC: Radiosurgery for spinal metastases: clinical experience in 500 cases from a single institution. Spine 2007, 32(2):193-199.

18. Chang EL, Shiu AS, Mendel E, Mathews LA, Mahajan A, Allen PK, Weinberg JS, Brown BW, Wang XS, Woo SY, et al: Phase I/II study of stereotactic body radiotherapy for spinal metastasis and its pattern of failure. J Neurosurg Spine 2007, 7(2):151-160.

19. Yamada Y, Bilsky MH, Lovelock DM, Venkatraman ES, Toner S, Johnson J, Zatcky J, Zelefsky MJ, Fuks Z: High-dose, single-fraction image-guided intensity-modulated radiotherapy for metastatic spinal lesions. Int J Radiat Oncol Biol Phys 2008, 71(2):484-490.

20. Sahgal A, Ames C, Chou D, Ma L, Huang K, Xu W, Chin C, Weinberg V, Chuang $C$, Weinstein $P$, et al: Stereotactic body radiotherapy is effective salvage therapy for patients with prior radiation of spinal metastases. Int J Radiat Oncol Biol Phys 2009, 74(3):723-731.

21. Gibbs IC, Patil C, Gerszten PC, Adler JR Jr, Burton SA: Delayed radiationinduced myelopathy after spinal radiosurgery. Neurosurgery 2009, 64(2):A67-72.

22. Nieder C, Grosu AL, Andratschke NH, Molls M: Proposal of human spinal cord reirradiation dose based on collection of data from 40 patients. Int J Radiat Oncol Biol Phys 2005, 61(3):851-855.

23. Ryu S, Jin JY, Jin R, Rock J, Ajlouni M, Movsas B, Rosenblum M, Kim JH: Partial volume tolerance of the spinal cord and complications of singledose radiosurgery. Cancer 2007, 109(3):628-636.

24. Sahgal A, Ma L, Gibbs I, Gerszten PC, Ryu S, Soltys S, Weinberg V, Wong S, Chang E, Fowler J, et al: Spinal cord tolerance for stereotactic body radiotherapy. Int J Radiat Oncol Biol Phys 2010, 77(2):548-553.

25. Nelson JW, Yoo DS, Sampson JH, Isaacs RE, Larrier NA, Marks LB, Yin FF, Wu QJ, Wang Z, Kirkpatrick JP: Stereotactic body radiotherapy for lesions of the spine and paraspinal regions. Int J Radiat Oncol Biol Phys 2009, 73(5):1369-1375.

26. Nguyen QN, Shiu AS, Rhines LD, Wang H, Allen PK, Wang XS, Chang EL: Management of spinal metastases from renal cell carcinoma using stereotactic body radiotherapy. Int J Radiat Oncol Biol Phys 2010, 76(4):1185-1192.

27. Mizumoto M, Harada H, Asakura H, Hashimoto T, Furutani $K$, Hashii H, Takagi T, Katagiri H, Takahashi M, Nishimura T: Prognostic factors and a scoring system for survival after radiotherapy for metastases to the spinal column: a review of 544 patients at Shizuoka Cancer Center Hospital. Cancer 2008, 113(10):2816-2822.

28. Guckenberger M, Goebel J, Wilbert J, Baier K, Richter A, Sweeney RA, Bratengeier K, Flentje M: Clinical outcome of dose-escalated image-guided radiotherapy for spinal metastases. Int J Radiat Oncol Biol Phys 2009, 75(3):828-835.

29. Chow E, Hoskin P, Mitera G, Zeng L, Lutz S, Roos D, Hahn C, van der Linden $Y$, Hartsell W, Kumar E: Update of the international consensus on 
palliative radiotherapy endpoints for future clinical trials in bone metastases. Int J Radiat Oncol Biol Phys 2012, 82(5):1730-1737.

30. Eisenhauer EA, Therasse P, Bogaerts J, Schwartz LH, Sargent D, Ford R, Dancey J, Arbuck S, Gwyther S, Mooney M, et al: New response evaluation criteria in solid tumours: revised RECIST guideline (version 1.1). Eur J Cancer 2009, 45(2):228-247.

31. Klish DS, Grossman P, Allen PK, Rhines LD, Chang EL: Irradiation of spinal metastases: should we continue to include one uninvolved vertebral body above and below in the radiation field? Int I Radiat Oncol Biol Phys 2011, 81(5):1495-1499.

doi:10.1186/1471-2407-12-530

Cite this article as: Guckenberger et al: Fractionated radiosurgery for painful spinal metastases: DOSIS - a phase II trial. BMC Cancer 2012 12:530.

\section{Submit your next manuscript to BioMed Central and take full advantage of:}

- Convenient online submission

- Thorough peer review

- No space constraints or color figure charges

- Immediate publication on acceptance

- Inclusion in PubMed, CAS, Scopus and Google Scholar

- Research which is freely available for redistribution 